REPRESENTACIONES SOCIALES FRENTE A LA

\title{
LACTANCIA MATERNA EN MUJERES RURALES Y URBANAS DE JALISCO, MÉXICO: ESTUDIO CUALITATIVO
}

\section{Sociall representations on breastfeeding among women living in urban and rural areas in Jalisco, Mexico: a quallitative study}

Verónica Guadalupe García-Magdaleno, $\mathrm{MSc}^{* 1}$; Jorge Laureano-Eugenio, $\mathrm{MSc}^{2}$

Recibido: enero 18/19 - Aceptado: junio 13/19

\section{RESUMEN}

Objetivo: comprender las representaciones sociales frente a la lactancia materna en mujeres de contextos rural y urbano, en Jalisco (México), entre 2016 y 2017.

Materiales y métodos: estudio cualitativo fenomenológico. En mujeres de comunidades rurales y urbanas de la Jurisdicción Sanitaria XII, Centro Tlaquepaque, Jalisco (México), identificadas por informantes clave, se realizaron entrevistas en grupos focales, grabadas en audio y analizadas semióticamente hasta llegar a la saturación teórica de las categorías: significado, actores generadores de significado y deseos frente a la lactancia materna.

Resultados: 6 grupos focales de 14 mujeres rurales, con edades de 29,4 \pm 7,8 años, y 9 mujeres urbanas con edades de 27,3 \pm 7,1 años. Cualitativamente, en ambos contextos se identificaron

\footnotetext{
Correspondencia: Verónica Guadalupe García Magdaleno, Secretaría de Salud. Departamento de Investigación. Dr. Baeza Alzaga \# 107 , Colonia: Centro, CP: 44100. Guadalajara, Jalisco.

Celular número: 0443329229101

licveronica.garcia.magdaleno@gmail.com

1 Maestra en Gestión de la Calidad en los Servicios de Salud; licenciada en Nutrición. O.P.D. Servicios de Salud, Jalisco (México).

2 Maestro en Ciencias de la Salud Pública; licenciado en Enfermería, Departamento de Investigación, Secretaría de Salud, Jalisco (México). georgelaure_1@hotmail.com
}

significados que se categorizaron en mitos, técnica de amamantamiento y crecimiento y desarrollo del lactante. Como actores generadores de significados se encontraron: personal de salud y hospital, red social, familia, Dios y comunidad. Como deseos las mujeres describen beneficios propios por amamantar y para el infante.

Conclusiones: las representaciones sociales frente a la lactancia materna tienen diferencias y similitudes de acuerdo con el contexto geográfico donde se desarrollan; los elementos y actores que las configuran determinan el inicio, mantenimiento o abandono de la lactancia y deben considerarse en las intervenciones de promoción y educación de la salud dirigidas a las mujeres y su entorno.

Palabras clave: lactancia materna; antropología cultural; análisis cualitativo.

\section{ABSTRACT}

Objective: To understand the social representations regarding breastfeeding among women in rural and urban settings in Jalisco (Mexico), between 2016 and 2017.

Materials and methods: Qualitative phenomenological study. Women living in rural and urban communities in the XIIth Health Jurisdiction, 
Tlaquepaque Center, Jalisco (Mexico), identified through key informants, were interviewed in focus groups, audio-recorded and semiotically analyzed until the technical saturation was reached in the following categories: meaning, meaning-generating players, and wishes pertaining to breastfeeding.

Results: Six focus groups of 14 women living in rural areas, aged $29.4 \pm 7.8$ years, and 9 women living in urban areas, aged $27.3 \pm 7.1$ años. Qualitatively, meanings were identified in the two settings and were categorized as myths, breastfeeding techniques, and infant growth and development. The following meaning-generating players were found: healthcare and hospital staff, social network, family, God, and community. In terms of wishes, women described the benefits of breastfeeding for themselves and their infants.

Conclusions: There are differences and similarities in social representations regarding breastfeeding depending on the geographic context where they are developed; the elements and players that make them up determine the initiation, maintenance or termination of breastfeeding, and must be considered as part of health promotion and education targeted to women and their environment.

Key words: breastfeeding; cultural anthropology; qualitative analysis.

\section{INTRODUCCIÓN}

La lactancia materna está posicionada por diversos organismos internacionales como un elemento clave en la alimentación del menor de edad (1), y está recomendada por la Organización Mundial de la Salud (OMS), de manera exclusiva durante los primeros 6 meses y complementaria hasta los 2 años de edad o más si la madre y el niño así lo desean $(2,3)$. Se le atribuyen beneficios como disminución de riesgo de atopías, otitis, neumonía, diarrea y enterocolitis (1, 4-6); está asociada a menor incidencia de obesidad y otras enfermedades crónicas. Su práctica contribuye a menor gasto de bolsillo familiar (3), regulación del ciclo hambre-saciedad y apego adecuado del infante; en la madre reduce el riesgo de cáncer de mama y de ovario, así como la osteoporosis (4). A pesar de lo anterior, se ha identificado que la población en diversos países ha incrementado el abandono de la lactancia materna sustituyéndola por sucedáneos de leche humana o práctica mixta (lactancia y sucedáneos). A nivel mundial, en la población de $0 \mathrm{a}$ 6 meses, la tasa de lactancia materna exclusiva es de $40 \%$ (2); en América Latina, específicamente en Colombia, 46,8\% y en Brasil, 38,6\% (7). En México, según Encuesta Nacional de Salud y Nutrición (Ensanut 2012), la duración de la lactancia materna es de 10 meses en promedio; en la zona rural se identifica que en menores de 6 meses es exclusiva en 18,5\% y en la zona urbana, 14,5\%. En ambos, 35,5\% continúa hasta el año y 14,1\% hasta los 2 años de vida (8).

Por su parte, las representaciones sociales como modalidad particular del conocimiento (9) cumplen la función de elaborar los comportamientos y la comunicación entre individuos (10), con lógica (sentido), lenguaje y derechos propios para el descubrimiento y la organización de la realidad; asimismo, dependen del aprendizaje, las creencias, los valores, las ideas, las normas y las prácticas socioculturales que permiten a los individuos orientarse en su mundo material y su cotidianidad (11). En este sentido, las acciones que buscan promover la lactancia materna deben considerar las características sociales, culturales y políticas (12) de cada contexto geográfico, debido a que es en este espacio donde las mujeres desarrollan su vida, generan relaciones familiares y comunitarias, establecen comunicación con los servicios de salud y reciben información, elementos que configuran sus representaciones sociales (13) para la toma de decisiones frente al inicio, mantenimiento o abandono de la lactancia materna.

Con el interés de optimizar esta práctica desde las instituciones de salud, es importante realizar un acercamiento metodológico para identificar y comprender la influencia que puede ejercer el contexto social, en particular las zonas rural o urbana, ya que investigaciones cuantitativas señalan que un 
menor índice de educación, situación económica desfavorable y poco acceso a la información de las mujeres rurales, a diferencia de las mujeres urbanas, condicionan que tengan una lactancia materna menos apropiada (14).

Por lo anterior, la Secretaría de Salud Jalisco, en busca de mejorar las acciones de educación y promoción de la salud, desarrolló una investigación cualitativa que se planteó el objetivo de comprender las representaciones sociales frente a la lactancia materna en mujeres de Jalisco, México, entre 2016 y 2017; en el en el presente artículo se muestran los principales resultados.

\section{MATERIALES Y MÉTODOS}

Diseño y población. Estudio cualitativo, desde los supuestos teóricos y metodológicos de la fenomenología, que permite explorar la manera en que las mujeres representan, aprenden y socializan la práctica de la lactancia materna, considerando que los significados configurados de manera subjetiva solo se comprenden a partir de la experiencia y la vivencia de las mujeres (15).

El universo de estudio fueron mujeres beneficiarias del programa Prospera (Programa de Desarrollo Social dirigido a personas en situación de pobreza, que busca ampliar las capacidades en alimentación, salud, educación y otras dimensiones de bienestar) que viven en comunidades rurales y urbanas de la Jurisdicción Sanitaria XII Centro Tlaquepaque, Jalisco (México), una de 13 jurisdicciones en la Secretaría de Salud.

Para la selección de mujeres de ambos contextos (rural y urbano) se partió del total de unidades de salud que conforman la Jurisdicción Sanitaria, de este listado se eligió, por muestreo aleatorio simple, una mujer rural y una urbana.

En estas primeras unidades de salud, para la selección de las mujeres se recurrió al muestreo teórico cualitativo, teniendo como perfil sociodemográfico los siguientes aspectos: a) más de 5 años como beneficiarias del programa Prospera, b) más de 5 años de vivir en la localidad, c) embarazadas o con hijos menores de un año. Se estimó un promedio de mínimo 4 informantes y máximo 6 por cada centro de salud con la finalidad de aplicar la técnica elegida.

Para definir el tamaño de la muestra se recurrió a la saturación teórica de la información, es decir, hasta el punto en que ya no se obtiene nueva información o esta comienza a ser redundante, de acuerdo con los ejes planteados para investigar: 1) significado de la lactancia materna, 2) actores generadores de significados frente a la práctica de la lactancia materna, 3) deseos de las mujeres frente a la lactancia materna; asimismo, para los investigadores fue importante la identificación de otras categorías emergentes dentro del trabajo de campo, sobre todo aquello que no se consideró al plantear la investigación (16).

Según lo anterior, se analizó la primera información y los investigadores decidieron elegir nuevamente, de manera aleatoria, otras unidades de salud, una rural y una urbana, y procedieron a seleccionar a las mujeres participantes a partir de los planteamientos del muestreo teórico utilizado anteriormente. Con el análisis de los grupos de estas segundas unidades de salud se llegó a la saturación teórica.

Procedimiento. El estudio se inició en octubre del 2016 y se dio por terminado en febrero del 2017; considerando que los dos investigadores (una nutrióloga y un enfermero) no tenían ninguna relación previa con las informantes y uno de ellos tampoco con el programa, identificaron y se entrevistaron con informantes clave de las unidades de salud por abordar: cuatro médicos, un trabajador social, un promotor de salud y tres enfermeras, quienes una vez que conocieron el objetivo del estudio y a partir de los criterios de muestreo teórico establecido invitaron personalmente a las mujeres a participar en la investigación.

Para recolectar la información se recurrió a la técnica de grupo focal, los dos investigadores tenían conocimientos y experiencias en su utilización. Las entrevistas se realizaron en aulas o espacios fuera de la unidad de salud, gestionados por los informantes 
clave. En cada grupo focal, un investigador (mujer) dirigía al grupo aplicando una guía de entrevista y otro investigador (hombre) participaba como observador no participante haciendo anotaciones. Se contó con la participación de una psicóloga para abordar situaciones en que se considerara necesario durante la entrevista.

La entrevista inició con la pregunta: ¿qué viene a su mente cuando escucha las palabras lactancia materna?, a partir de esta se permitió a las informantes de cada grupo iniciar un proceso de diálogo, pasando a la indagación de los ejes planteados para la investigación. En cada grupo focal se empleó un tiempo promedio de 1 hora 30 minutos. Para la documentación de la información, se realizó grabación de audio, con previa autorización y consentimiento por escrito de las informantes. Cabe mencionar que ninguna informante desistió de participar en ningún momento del estudio, y que al llegar al punto de saturación no hubo necesidad de realizar más grupos focales.

Análisis. Para lograr la confiabilidad de los datos se consideraron diversos aspectos citados por algunos autores: a) mantener una observación persistente y prolongada durante todo el trabajo, b) se citaron otros estudios realizados y la normatividad en el tema al inicio y al final del trabajo, c) se devolvieron los resultados a un grupo de mujeres informantes, convocadas por informantes clave para corroboración y afirmación de los hallazgos plasmados, d) se comprobó la saturación de datos por los dos investigadores (17).

Las grabaciones de las entrevistas se transcribieron a textos de word y se analizaron bajo el modelo actancial semiótico, lo que implicó la lectura y relectura de cada línea textual; la descripción de primeras impresiones contextualizadas, tanto de ejes considerados como de temas emergentes; el análisis de la superficie de los textos; la identificación de actores semióticos, isotopías temáticas y figurativas, dimensión cognitiva, así como relaciones ideológicas recíprocas en las que se pueden identificar deseos, generó una estructuración del texto en grandes categorías con descripciones textuales que documentaran la perspectiva de las mujeres frente a la práctica de la lactancia materna $(18,19)$.

Aspectos éticos. La participación de las mujeres en el estudio fue voluntaria y bajo consentimiento informado por escrito, respetando su autonomía para participar, autodeterminación y confidencialidad de la información; además, el protocolo fue dictaminado por el Comité de Investigación y el Comité de Ética en Investigación de la Oficina Central de la Secretaría de Salud Jalisco, en cumplimiento de los criterios métodológicos, éticos y de originalidad, de acuerdo con la Norma Técnica 313; se obtuvo el registro 35/RXII-JAL/2016.

\section{RESULTADOS}

En cuatro unidades de salud se realizaron un total de 6 entrevistas grupales, 3 en cada contexto, con un total de 23 informantes: $13 \%(n=3)$ estaban embarazadas y $87 \%(n=20)$ tenía hijos menores de 1 año. Del total, $61 \%(\mathrm{n}=14)$ vivían en contexto rural, con una media de 29,4 años (desviación estándar DE $\pm 7,8$ ) y una mediana de 4 hijos (rango: $1-6)$; de estas mujeres el $93 \%(n=13)$ se encontraba practicando lactancia o estaba a favor de hacerlo. El resto, 39\% (n=9) eran del contexto urbano, con una media de edad 27,3 años (DE $\pm 7,1)$ y una mediana de 4 hijos (rango: 2- 5); de ellas, el $100 \%$ $(n=9)$ estaba a favor de la práctica. Las características socio-demográficas de las participantes se muestran en la tabla 1.

Según los ejes de investigación, los hallazgos se clasificaron en significados, actores y deseos frente a la práctica de la lactancia materna.

Significados frente a la práctica de la lactancia materna: en el discurso se identifican y describen elementos significativos con diferencias y similitudes por contextos (tabla 2).

Actores generadores de significados frente a la práctica de la lactancia materna: en la representación social de las mujeres también hay actores que contribuyen a dar el significado y sentido de actuar que tienen las mujeres. Estos actores se tipifican y describen en 


\begin{tabular}{|c|c|c|c|c|c|}
\hline \multirow{2}{*}{\multicolumn{2}{|c|}{ Variables }} & \multicolumn{2}{|c|}{ Contexto rural } & \multicolumn{2}{|c|}{ Contexto urbano } \\
\hline & & fr & $\%$ & fr & $\%$ \\
\hline \multirow{3}{*}{ Estado civil } & Casada & 10 & 71 & 6 & 67 \\
\hline & Unión libre & 4 & 29 & 2 & 22 \\
\hline & Soltera & 0 & 0 & 1 & 11 \\
\hline \multirow{4}{*}{ Estudios } & Primaria & 2 & 14 & 1 & 11 \\
\hline & Secundaria & 9 & 64 & 5 & 56 \\
\hline & Preparatoria & 3 & 21 & 2 & 22 \\
\hline & Licenciatura & 0 & 0 & 1 & 11 \\
\hline \multirow{2}{*}{ Profesión u ocupación } & Hogar & 13 & 93 & 8 & 89 \\
\hline & Empleada & 1 & 7 & 1 & 11 \\
\hline \multirow{2}{*}{ Religión } & Católica & 14 & 100 & 8 & 89 \\
\hline & Sin religión & 0 & 0 & 1 & 11 \\
\hline
\end{tabular}

Fuente: trabajo de campo.

la tabla 3. Ante la descripción del actante comunidad, entre las mujeres del contexto rural prevalece una posición de empoderamiento frente al enjuiciamiento, tal como se representa en el siguiente testimonio: "pues es nuestro derecho, ¿por qué se meten en eso?". No obstante, en otras mujeres sí genera influencia: "No, pues'ta mal darle [lactancia] en público porque al rato te critican”.

Deseos de las mujeres frente a la lactancia materna: en las representaciones sociales de las informantes de este estudio se destacan dos deseos, en el primero, la mujer que amamanta reconoce querer beneficiarse ella misma, con diferentes elementos de influencia que se describen en la figura 1. Estos beneficios se observan en los siguientes testimonios: "Te ahorras mucho dinero", "para tener nuestro cuerpo como antes", "vínculo estrecho que tenemos las mamás" y "te limpia el pecho, te previene el cáncer de mamá". Cabe mencionar que además de los beneficios, en el discurso también se observaron perjuicios, clasificados en las barreras: “... porque también a veces no quieren dar [lactancia] porque dicen que se les hacen aguadas". En el segundo deseo, las mujeres esperan beneficiar al lactante en cuestiones nutricionales, psicológico-afectivas, de crecimiento, desarrollo y mejoras en salud, aspectos que se expresan así: "Son las mejores vitaminas que puedes tener para tu bebé", "les das más seguridad, porque se sienten protegidos", "les ayuda... a que crezcan más fuertes y se desarrollen más", "no va a tener ninguna enfermedad". Finalmente, es importante señalar que durante las entrevistas no se generaron categorías emergentes.

\section{DISCUSIÓN}

Entre los significados encontrados en este estudio se destacan mitos, características de los senos y de la leche materna, lesiones por técnica de amamantamiento e influencia en el crecimiento y desarrollo del lactante. Actores como el personal de salud, el hospital, la red social Facebook, la familia, Dios y la comunidad son identificados como aquellos que contribuyen a la toma de decisiones. Sobre técnicas de amamantamiento, en específico de agarre 


\begin{tabular}{|c|c|c|c|c|c|}
\hline \multicolumn{6}{|c|}{$\begin{array}{c}\text { Tabla } 2 . \\
\text { Elementos significativos frente a la práctica de la lactancia en mujeres rurales y urbanas, } \\
\text { Jalisco, México, } 2017\end{array}$} \\
\hline \multicolumn{3}{|c|}{ Contexto rural } & \multicolumn{3}{|c|}{ Contexto urbano } \\
\hline Categoría & Descripción & $\begin{array}{l}\text { Texto } \\
\text { representativo }\end{array}$ & Categoría & $\begin{array}{l}\text { Descrip- } \\
\text { ción }\end{array}$ & $\begin{array}{l}\text { Texto } \\
\text { representativo }\end{array}$ \\
\hline $\begin{array}{l}\text { Mitos } \\
\text { (condicionantes } \\
\text { en lactancia) }\end{array}$ & $\begin{array}{l}\text { Las informantes aluden } \\
\text { que menor edad equi- } \\
\text { vale a menor experien- } \\
\text { cia para establecer la } \\
\text { lactancia }\end{array}$ & $\begin{array}{l}\text { “Quería él [bebé] } \\
\text { agarrarme el pecho } \\
\text { y a parte que uno } \\
\text { está mocoso y no } \\
\text { sabe ni que, [el bebé] } \\
\text { no quiso no quiso y } \\
\text { ya no le rogué ni } \\
\text { nada” }\end{array}$ & \multirow[t]{4}{*}{$\begin{array}{l}\text { Técnica } \\
\text { de amaman- } \\
\text { tamiento } \\
\text { (agarre del } \\
\text { seno) }\end{array}$} & \multirow{4}{*}{$\begin{array}{l}\text { Las mujeres } \\
\text { mencio- } \\
\text { naron que } \\
\text { sentir dolor } \\
\text { y tener } \\
\text { heridas en } \\
\text { los senos } \\
\text { son motivo } \\
\text { para elegir } \\
\text { otra forma } \\
\text { de alimenta- } \\
\text { ción }\end{array}$} & \multirow[t]{4}{*}{$\begin{array}{l}\text { "No aguanté } \\
\text { porque me } \\
\text { dolía y aparte } \\
\text { me sangraban } \\
\text { las dos... mejor } \\
\text { le doy biberón } \\
\text { de leche" }\end{array}$} \\
\hline $\begin{array}{l}\text { Mitos } \\
\text { (características } \\
\text { de los senos) }\end{array}$ & $\begin{array}{l}\text { Las expresiones sugie- } \\
\text { ren que el tamaño de } \\
\text { los senos y la variedad } \\
\text { anatómica del pezón } \\
\text { condicionan el ama- } \\
\text { mantamiento }\end{array}$ & $\begin{array}{l}\text { “... [las mujeres] } \\
\text { porque tienen chichis } \\
\text { chiquitas o que no } \\
\text { tienen el pezón... no } \\
\text { chupa el bebé y si no } \\
\text { chupa el bebé pues [la } \\
\text { leche] no te sale” }\end{array}$ & & & \\
\hline $\begin{array}{l}\text { Crecimiento y } \\
\text { desarrollo del } \\
\text { lactante }\end{array}$ & $\begin{array}{l}\text { Los dientes y la habili- } \\
\text { dad de morder conlle- } \\
\text { van reacciones frente } \\
\text { al mantenimiento de la } \\
\text { lactancia materna }\end{array}$ & $\begin{array}{l}\text { “...[Al bebé] le salie- } \\
\text { ron los dientes y yo } \\
\text { no quería porque me } \\
\text { mordía, me dolía, pero } \\
\text { no le dejé de dar por } \\
\text { eso” }\end{array}$ & & & \\
\hline $\begin{array}{l}\text { Técnica de } \\
\text { amamantamiento } \\
\text { (agarre del seno) }\end{array}$ & $\begin{array}{l}\text { Las informantes } \\
\text { señalan que cuando la } \\
\text { mujer que amamanta } \\
\text { tiene pezón plano o } \\
\text { invertido, se procura el } \\
\text { agarre alternativo }\end{array}$ & $\begin{array}{l}\text { “... vi una mucha- } \\
\text { cha... no tenía pezón } \\
\text { y le amarraron una liga } \\
\text { pa 'que se le hiciera... } \\
\text { y el bebé chupara” }\end{array}$ & & & \\
\hline \multicolumn{6}{|c|}{ Ambos contextos } \\
\hline \multicolumn{2}{|c|}{ Categoría } & Descripción & \multicolumn{3}{|c|}{ Texto representativo } \\
\hline $\begin{array}{l}\text { Mitos } \\
\text { (condicionantes e }\end{array}$ & lactancia) & $\begin{array}{l}\text { Se percibe que no te- } \\
\text { ner experiencia previa } \\
\text { en amamantamiento } \\
\text { limita los conocimien- } \\
\text { tos sobre la lactancia } \\
\text { materna }\end{array}$ & \multicolumn{3}{|c|}{$\begin{array}{l}\text { "Apenas es mi primer bebé, es muy poco lo } \\
\text { que sé" }\end{array}$} \\
\hline $\begin{array}{l}\text { Mitos } \\
\text { (leche materna) }\end{array}$ & & $\begin{array}{l}\text { Las mujeres creen que } \\
\text { la leche materna es un } \\
\text { medio de transmisión } \\
\text { de enfermedades }\end{array}$ & \multicolumn{3}{|c|}{$\begin{array}{l}\text { “...una hermana mía dejó de lactar... le } \\
\text { dijeron no puedes darle porque lo puedes } \\
\text { contaminar a tu bebé, transmitirle esa enfer- } \\
\text { medad... es un método de transmisión de } \\
\text { enfermedades” }\end{array}$} \\
\hline
\end{tabular}

Fuente: trabajo de campo. 


\begin{tabular}{|c|c|c|c|c|c|}
\hline Actores ge & lores de significa & $\begin{array}{r}\text { Tabla } 3 . \\
\text { s frente a la práctica } \\
\text { Jalisco, México, }\end{array}$ & $\begin{array}{l}\text { le la lact } \\
2017\end{array}$ & cia, en mujeres ru & les y urbanas, \\
\hline & Contexto rur & & & Contexto urba & \\
\hline Actor & Descripción & $\begin{array}{l}\text { Texto } \\
\text { representativo }\end{array}$ & Actor & Descripción & $\begin{array}{l}\text { Texto } \\
\text { representativo }\end{array}$ \\
\hline $\begin{array}{l}\text { Personal } \\
\text { médico }\end{array}$ & $\begin{array}{l}\text { Personaje de } \\
\text { contraste, que } \\
\text { determina la } \\
\text { práctica de la } \\
\text { lactancia materna }\end{array}$ & $\begin{array}{l}\text { "Dijo que sí le tenía } \\
\text { que dar pecho y } \\
\text { después que no le caía } \\
\text { porque era bien mulo" }\end{array}$ & Familia & $\begin{array}{l}\text { Madre es la mu- } \\
\text { jer cercana que le } \\
\text { transmite experien- } \\
\text { cias y saberes frente } \\
\text { a la práctica de la } \\
\text { lactancia }\end{array}$ & $\begin{array}{l}\text { "A través de mi } \\
\text { madre, que, } \\
\text { como vi que } \\
\text { amamantó a mis } \\
\text { hermanos... } \\
\text { sobre ella sigo } \\
\text { orientándome } \\
\text { ahora ya que me } \\
\text { casé" }\end{array}$ \\
\hline $\begin{array}{l}\text { Personal de } \\
\text { enfermería }\end{array}$ & $\begin{array}{l}\text { Facilitador de } \\
\text { información en el } \\
\text { periodo prenatal }\end{array}$ & $\begin{array}{l}\text { "Enfermería cuando } \\
\text { uno está embarazada... } \\
\text { citan a las embaraza- } \\
\text { das... para hacer eso, } \\
\text { [hablar]: cómo le vas } \\
\text { a hacer, cómo le vas } \\
\text { a dar, posición, cómo } \\
\text { darle" }\end{array}$ & Dios & $\begin{array}{l}\text { Deidad a la que } \\
\text { se atribuyen los } \\
\text { beneficios de la } \\
\text { leche materna }\end{array}$ & $\begin{array}{l}\text { "La lactancia es } \\
\text { buena porque } \\
\text { Dios la hizo" }\end{array}$ \\
\hline Hospital & $\begin{array}{l}\text { Institución que } \\
\text { promueve la alimen- } \\
\text { tación exclusiva con } \\
\text { leche materna }\end{array}$ & $\begin{array}{l}\text { "Ya hasta los hospitales } \\
\text { no dejan que uno les dé } \\
\text { bibí, ya lo prohibieron, } \\
\text { hay muchos carteles de } \\
\text { puro pecho, el pecho es } \\
\text { lo mejor" }\end{array}$ & & & \\
\hline $\begin{array}{l}\text { Red social } \\
\text { Facebook }\end{array}$ & $\begin{array}{l}\text { Medio de comuni- } \\
\text { cación donde se ob- } \\
\text { serva la práctica de } \\
\text { la lactancia materna }\end{array}$ & $\begin{array}{l}\text { "En el face... he } \\
\text { visto... se han hecho } \\
\text { huelgas de personas... } \\
\text { de mamás que se sien- } \\
\text { tan ahí en público y casi } \\
\text { desnudas, pues casi sin } \\
\text { blusa, así dándoles de } \\
\text { comer" }\end{array}$ & & & \\
\hline & & Ambos contex & & & \\
\hline & Actor & Descripción & & Texto repre & entativo \\
\hline Comunidad & & $\begin{array}{l}\text { Personaje que según las i } \\
\text { tes realiza enjuiciamientc } \\
\text { práctica pública de la lac }\end{array}$ & $\begin{array}{l}\text { s sorman- } \\
\text { ancia }\end{array}$ & $\begin{array}{l}\text { "Un señor le dijo a n } \\
\text { le estuviera dando [la } \\
\text { [de él]" }\end{array}$ & $\begin{array}{l}\text { i cuñada que no } \\
\text { tancia] en frente }\end{array}$ \\
\hline
\end{tabular}

Fuente: trabajo de campo. 


\section{Figura 1.}

Beneficio, facilitadores y barreras percibidas por las mujeres frente a la práctica de la lactancia, en mujeres rurales y urbanas, Jalisco, México, 2017

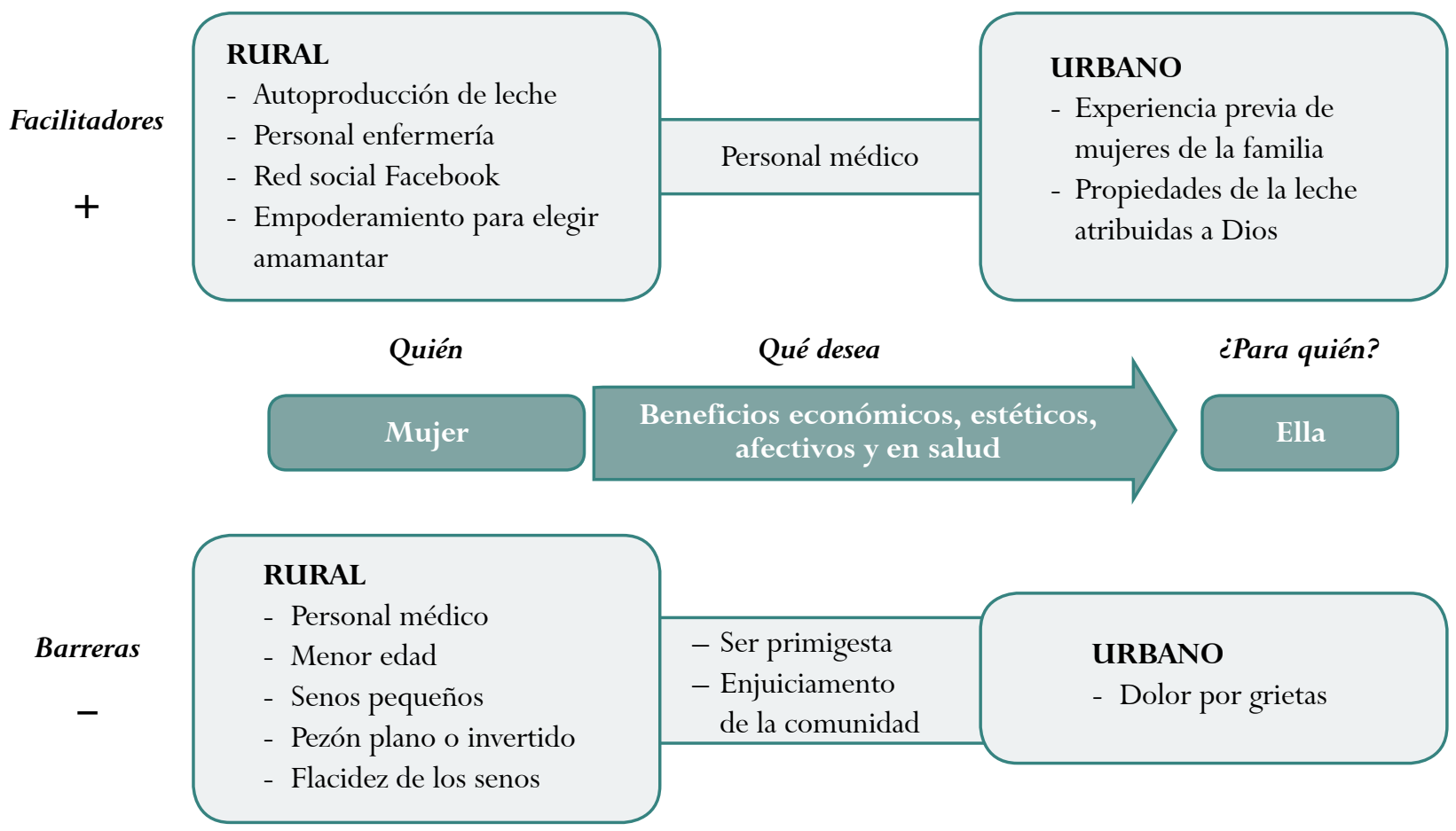

del seno, los inconvenientes expresados (mordidas, grietas) por las informantes en este estudio como barreras para mantener la lactancia también se han documentado en otras investigaciones en México y América Latina $(20,21)$, lo que fundamenta el fortalecimiento de promoción y difusión de información relacionada con el crecimiento y desarrollo de los infantes y la lactancia, así como la técnica correcta de amamantamiento.

Otro elemento clave considerado en las representaciones sociales es la información recibida en el contexto rural, donde se asume que la que proporcionan los profesionales de la salud (médico y enfermería) es información creíble y decisiva en torno al amamantamiento. Esto contrasta con lo descrito en otras investigaciones donde se refiere que la falta de recomendaciones y apoyo del personal de salud tiene relación negativa con la lactancia $(22,23)$. Lo anterior evidencia la necesidad de posicionar a otros profesionales de la salud (nutriólogos, trabajadores sociales y otros) y de brindar capacitación continua y acompañamiento por expertos que contribuyan a la transmisión de conocimientos adecuados, desmitificación oportuna y desarrollo de aptitudes y actitudes necesarias para fomentar y proteger la lactancia materna. También es relevante destacar que el "hospital" fue la única institución de salud mencionada por las mujeres en este estudio como promotora de lactancia, lo que evidencia la falta de posicionamiento que tienen las unidades de salud como instituciones de primer contacto en esta materia.

Las mujeres del contexto rural señalaron a la red social Facebook como un proveedor de conocimientos; otra investigación similar refiere que en los medios digitales efectivamente se puede ilustrar la creación de ideologías y prácticas sobre la alimentación al pecho (12), por tanto, sería conveniente que el sector salud considere la incorporación de 
estrategias de difusión, comunicación y conocimientos verídicos en torno a la lactancia en este tipo de medios (redes sociales electrónicas).

La descripción de Dios como actor identificado en este estudio en el contexto urbano, se asemeja a lo referido en otra investigación donde mujeres del mismo contexto hacen atribuciones divinas a la experiencia única del amamantamiento relacionada con una bendición otorgada por esta deidad (22), aspecto ideológico relevante que se debe considerar en los acercamientos a la población para la generación de comunicación empática que logre afianzar las relaciones con los proveedores de salud. También se ha documentado que la familia (madre) es un elemento de apoyo e influencia en la decisión de dar pecho, por tanto, es conveniente ampliar las acciones de promoción que incluyan a familiares cercanos y a la pareja, que aunque no fue mencionada en el presente estudio, también se ha evidenciado como soporte para la continuidad de la lactancia $(13,24,25)$. Por otro lado, según lo referido, las mujeres de ambos contextos perciben falta de normalidad comunitaria para el amamantamiento público, obstáculo ya reconocido como limitante para el éxito de la lactancia en otras publicaciones (26) que refleja la necesidad de trabajar en prácticas y legislación que conlleven la normalización de esta práctica como un acto y un derecho natural y benéfico de alimentación en la infancia.

En cuanto a deseos identificados, hay similitud entre lo descrito en este estudio y otras investigaciones, tanto en los múltiples beneficios $(1,4-6)$ como en los perjuicios estéticos (flacidez de los senos) (26, 27) que las mujeres perciben para sí mismas. Lo mismo ocurre con el deseo de beneficiar al infante amamantado por medio del vínculo emocional y en favor del crecimiento y desarrollo $(22,26)$, lo que ofrece la oportunidad de brindar mayor información sobre otros beneficios en torno a la práctica que no fueron mencionados en estos resultados.

Finalmente, cabe destacar que todos los aspectos sociales y culturales vividos y expresados en torno a la lactancia deben considerarse y respetarse dentro del derecho de atención a la promoción de la salud.

\section{CONCLUSIONES}

Las representaciones sociales de las mujeres frente a la lactancia materna tienen diferencias y similitudes según el contexto geográfico donde se desarrollan, y son configuradas por elementos y actores que otorgan significado y determinan el inicio, mantenimiento o abandono de la lactancia. Algunos de ellos son percibidos como ayudas o como barreras para lograr los beneficios que la mujer que amamanta espera para sí misma y para el lactante.

\section{AGRADECIMIENTOS}

A las autoridades y al Departamento de Investigación de la Secretaría de Salud y O.P.D. Servicios de Salud Jalisco; a la doctora Tatiana de la Torre, a las licenciadas Johanna Vergara y Guadalupe Camarena, a los doctores Pavel Contreras y Carlos Ruiz Esparza Macías, a la maestra Xóchitl Fernández, al personal de las unidades de salud de la Jurisdicción Sanitaria XII Centro Tlaquepaque, y a todas las mujeres que con su participación ofrecieron la oportunidad de saber más allá de lo conocido.

\section{REFERENCIAS}

1. González N, López GA, Prado LM. Importancia de la nutrición: primeros 1000 días de vida. Acta Pediatr Hondu. 2016;7(1):597-607.

2. Organización Mundial de la Salud. Estrategia mundial para la alimentación del lactante y el niño pequeño. 54 AM de la Salud; 2001.

3. Organización Mundial de la Salud (OMS). Alimentación del lactante y del niño pequeño. Suiza: Organización Mundial de la Salud; 2018 [visitado 2018 may 22]. Disponible en: http://www.who.int/es/news-room/ fact-sheets/detail/infant-and-young-child-feeding

4. González de Cosío-Martínez T, Hernández-Cordero S, Rivera-Dommarco J, Hernández-Ávila M, Comité de Expertos. Recomendaciones para una política nacional de promoción de la lactancia materna en 
México: postura de la Academia Nacional de Medicina. Salud Pública Mex. 2017;59:106-13. http://dx.doi. org/10.21149/8102

5. García-López R. Composición e inmunología de la leche humana. Acta Pediatr Mex. 2011;32(4):223-30.

6. Urquizo Arístegui R. Lactancia materna exclusiva: ¿siempre? Rev Peru Ginecol Obstet. 2014 [visitado 2018 may 23];60(2):171-6. Disponible en: http://www.scielo.org.pe/scielo.php? script $=$ sci arttext\&pid =S2304-51322014000200011\&lng=es

7. Organización Panamericana de la Salud (OPS), Organización Mundial de la Salud (OMS). Oficina Regional de las Américas. Situación actual y tendencias de la lactancia materna en América Latina y el Caribe: implicaciones políticas y programáticas. Washington: OPS; 2012.

8. Gutiérrez JP, Rivera-Dommarco J, Shamah-Levy T, Villalpando-Hernández S, Franco A, Cuevas-Nasu L et al. Encuesta Nacional de Salud y Nutrición 2012. Resultados Nacionales. Cuernavaca: Instituto Nacional de Salud Pública (MX); 2012.

9. Villamañán M. Lo comunitario en las representaciones sociales de la violencia. Psicol Soc. 2016;28(3):494504. https://dx.doi.org/10.1590/1807-03102 016v $28 n 3 p 494$

10. Caravaca J. Las representaciones sociales y la cognición social: contribuciones para la investigación en enfermería y salud. Texto Contexto-Enferm. 2017;26(4):2-9. http://dx.doi.org/10.1590/010407072017001500017

11. Assunção M. Teoría de las representaciones sociales y contribuciones para las investigaciones sobre atención en salud y en enfermería. Esc Anna Nery. 2016;20(2):214-9.

12. Rodríguez R. Aproximación antropológica a la lactancia materna. Rev Antropol Exp. 2015;15(23):407-29.

13. Pérez MD, Moreno A. Dando voz a las mujeres: representaciones sociales y experiencias sobre lactancia. Doss Fem. 2017;22:107-17. http://dx.doi. org/10.6035/Dossiers.2017.22.7

14. Marín-Arias L, Ureña ME, Fernández X. Duración de la lactancia materna exclusiva en una comunidad urbana y otra rural de Costa Rica. Pob y Salud Mes. 2018;15(2):1-20. https://doi.org/10.15517/psm. v15i2.26408

15. Mercado FJ, Gastaldo D, Calderón C. Paradigmas y diseños de la investigación cualitativa en salud. Una antología Iberoamericana. México: Universidad de Guadalajara, Universidad Autónoma de Nuevo León, Servicio Vasco de Salud Osakidetza y Asociación Médica de Jalisco; 2002.

16. Martín C, Salamanca AC. El muestreo en la investigación cualitativa. Nure Investigación. 2007 [visitado 2017 feb 02];27. Disponible en: http://www.sc.ehu. es/plwlumuj/ebalECTS/praktikak/muestreo.pdf.

17. Santaella CM. Criterios de validez en la investigación cualitativa actual. Revista de Investigación Educativa. 2006 [visitado 2017 feb 02];24(1). Disponible en: http://revistas.um.es/rie/article/viewFile/97351/93461.

18. Karam T. Introducción a la semiótica. Portal de la Comunicación InCom-UAB: el portal de los estudios de comunicación, 2001-2011 [visitado 2017 feb 2]. Disponible en: http://www.portalcomunicacion.com/ uploads/pdf/18_esp.pdf.

19. Greimas AJ, Campodónico H, Courtés J, Ballón E. Semiótica: diccionario razonado de la teoría del lenguaje. Madrid: Gredos; 1990.

20. Laghi-Rey A, Yaipen-Ayca A, Risco-Denegri R, Pereyra-Elías R. Factores asociados al abandono de la lactancia materna exclusiva en una muestra de madres de Lima, Perú. Arch Med. 2013;11(3):1-7.

21. Espinoza C, Zamorano C, Graham S, Orozco A. Factores que determinan la duración de la lactancia materna durante los tres primeros meses de vida. An Med (Mex). 2014;59(2):120-6.

22. Gutiérrez-Obregón Y, Marín-Arias L. Significado de lactancia materna y leche materna para las madres de una comunidad urbana y otra rural de Costa Rica. Pob Salud Mes. 2017;15(1):4. 10.15517/psm. v15i1.26414

23. Castro A. Conocimiento y actitudes de los profesionales de la salud en la lactancia materna: traducción, validación y adaptación del cuestionario Child Care 
Providers Knowledge and Attitudes regarding support of breastfeeding. Nure Inv. 2018;15(92):1-9.

24. Becerra-Bulla F, Rocha-Calderón L, Fonseca-Silva DM, Bermúdez-Gordillo LA. El entorno familiar y social de la madre como factor que promueve o dificulta la lactancia materna. Rev Fac Med. 2015;63(2):217-27. http://dx.doi.org/10.15446/revfacmed.v63n2.44051

25. Valenzuela S, Vásquez E, Gálvez P. Factores que influyen en la disminución de lactancia materna exclusiva hasta los 6 meses de vida: revisión temática y contexto en Chile. Rev Int Salud Matern Fetal. 2016;1(7):12-9.
26. Díaz-Gómez NM, Ruzafa-Martínez M, Ares S, Espiga I, De Alba C. Motivaciones y barreras percibidas por las mujeres españolas en relación a la lactancia materna. Rev Esp Salud Pública. 2016 [visitado 2018 oct 23];90:e40016. Disponible en: http://scielo.isciii.es/scielo.php? script $=$ sci_ arttext\&pid $=\mathrm{S} 1135-57272016000100416 \& \operatorname{lng}=\mathrm{es}$

27. Carchi RG, León BC. Factores relacionados en la lactancia artificial en niños menores de 2 años [Tesis]. 2018 [visitado 2018 oct 11]. Disponible en: http:// dspace.ucuenca.edu.ec/handle/123456789/29311 